\title{
Development of high-performance whole cell biosensors aided by statistical modelling
}

\author{
Adokiye Berepiki, ${ }^{\dagger}$ Ross Kent, ${ }^{\dagger}$ Leopoldo F. M. Machado, ${ }^{\dagger}$ Neil Dixon ${ }^{\dagger \neq *}$ \\ †Manchester Institute of Biotechnology (MIB), ‡SYNBIOCHEM, Department of Chemistry, \\ University of Manchester, Manchester, M1 7DN, UK
}

\section{SUPPORTING INFORMATION}

\section{Contents}

Additional Methods................................p2

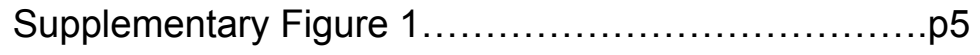

Supplementary Figure $2 \ldots \ldots \ldots \ldots \ldots \ldots \ldots \ldots \ldots \ldots \ldots$ p6

Supplementary Figure $3 \ldots \ldots \ldots \ldots \ldots \ldots \ldots \ldots \ldots \ldots . . . \ldots 7$

Supplementary Figure $4 \ldots \ldots \ldots \ldots \ldots \ldots \ldots \ldots \ldots \ldots . . p 8$

Supplementary Table $1 \ldots \ldots \ldots \ldots \ldots \ldots \ldots \ldots \ldots \ldots . . . . . . \ldots 9$

Supplementary Table $2 \ldots \ldots \ldots \ldots \ldots \ldots \ldots \ldots \ldots \ldots \ldots \ldots$

Supplementary Table $3 \ldots \ldots \ldots \ldots \ldots \ldots \ldots \ldots \ldots \ldots . . . \ldots 11$

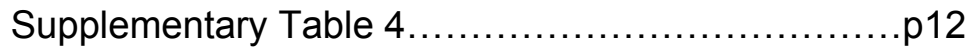

Supplementary Table $5 \ldots \ldots \ldots \ldots \ldots \ldots \ldots \ldots \ldots \ldots . . . . \ldots 13$

Supplementary Table $6 \ldots \ldots \ldots \ldots \ldots \ldots \ldots \ldots \ldots \ldots . . . \ldots \ldots$

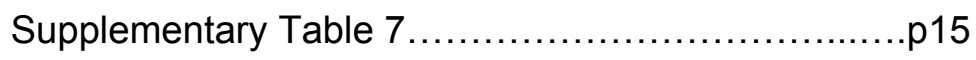

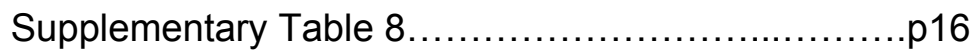

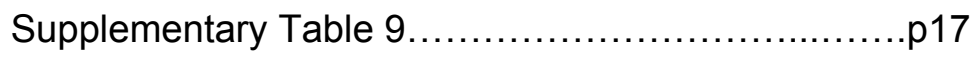

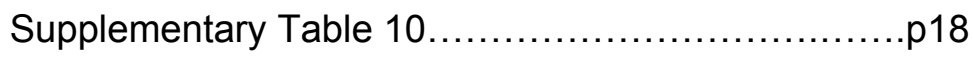

Supplementary Table $11 \ldots \ldots \ldots \ldots \ldots \ldots \ldots \ldots \ldots \ldots . . . \ldots 22$

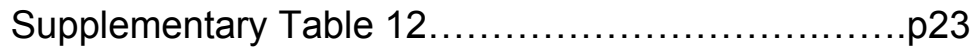

Supplementary Table $13 \ldots \ldots \ldots \ldots \ldots \ldots \ldots \ldots \ldots \ldots . . . \ldots 24$

Supplementary References..........................p25 


\section{Additional methods - Molecular cloning}

The PCA biosensor was assembled by isothermal assembly from the following fragments: pSEVA131 linearized by inverse PCR (primers AB9/10); mCherry amplified with primers $A B 15 / 28$ from a synthetic gene (GeneArt); sfGFP amplified with primers AB18/27 from a synthetic gene (GeneArt), and synthetic DNA (IDT) incorporating the ProB promoter (1) fused to a strong RBS (gaaataaggaggtaatacaa) (2), the $P_{P V}$ promoter (3) fused to the G10 RBS (4) and a 150 bp spacer (5) to yield the template plasmid (p131B). Promoter ( $P_{\text {reg-lib and } P_{\text {out }}-\text { lib) }}$ and RBS (RBS out lib) libraries were generated by linearising p131B by inverse PCR with

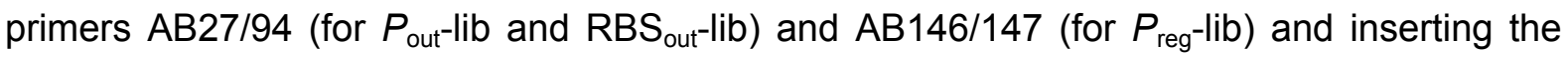
following degenerate ssDNA oligonucleotides via isothermal assembly: for $P_{\text {out }}$ lib oligo

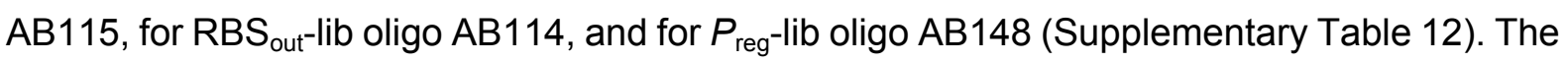
library members were designated p131B-BX for $P_{\text {reg-lib, p131B-GX for RBS }}$ out-lib, and p131$V X$ for $P_{\text {out }}$-lib, with $X$ denoting the clone number, which was assigned based on subsequent screening and rank order of expression output.

Constructs corresponding to the DoE Definitive Screening Design table (Table 1) were generated in three stages. Firstly, mCherry was replaced with pcaV using in vivo assembly, using the selected library plasmids (those with $P_{\text {reg }}$ at level $-1,0$ and +1 ) linearized by inverse PCR with primers $A B 10 / 128$, and pcaV amplified from pPv-Pcav (p44-pcaV) (3) with primers AB96/127, to yield pD2 $\left(P_{\text {reg }} / P_{\text {out }} / \mathrm{RBS}_{\text {out }}\right.$ pattern at level $\left.0 /+1 /+1\right), \mathrm{pD} 7\left(P_{\text {reg }} / P_{\text {out }} / \mathrm{RBS}_{\text {out }}\right.$ at levels $+1 /+1 /+1)$, and p131C-B20 $\left(P_{\text {reg }} / P_{\text {out }} / R_{B S}\right.$ out pattern at level $\left.-1 /+1 /+1\right)$. Secondly, these plasmids were again linearized by inverse PCR with primers $A B 27 / 94$ and the oligos $A B 142$, AB143, AB144 and AB145 (corresponding $P_{\text {out }} /$ RBS $_{\text {out }}$ patterns at level 0/-1, 0/0, -1/-1 and $1 / 0$, respectively), were inserted by isothermal assembly to create plasmids $\mathrm{pD} 1$ $\left(P_{\text {reg }} / P_{\text {out }} /\right.$ RBS $_{\text {out }}$ pattern at level $\left.0 / 0 / 0\right), \mathrm{pD} 3\left(P_{\text {reg }} / P_{\text {out }} / \mathrm{RBS}_{\text {out }}\right.$ pattern at level $\left.-1 /-1 /-1\right), \mathrm{pD} 4$ $\left(P_{\text {reg }} / P_{\text {out }} /\right.$ RBS $_{\text {out }}$ pattern at level $\left.+1 /-1 / 0\right)$, pD6 $\left(P_{\text {reg }} / P_{\text {out }} /\right.$ RBS $_{\text {out }}$ pattern at level $\left.0 /-1 /-1\right)$, and pD8 $\left(P_{\text {reg }} / P_{\text {out }} /\right.$ RBS $_{\text {out }}$ pattern at level $\left.+1 / 0 /-1\right)$. Next, the final set of DoE constructs were made by in vivo assembly using selected plasmids from the $P_{\text {out }}$-lib and RBS $_{\text {out }}$-lib libraries linearized by inverse PCR with primers AB10/130, and $P_{\text {reg }}-p c a V$ amplified with primers $A B 11 / 129$ from pD2, pD7 and p131C-B20. This yielded plasmids pD5 ( $P_{\text {reg }} / P_{\text {out }} / R_{\text {RSS }}$ out pattern at level $\left.-1 /+1 / 0\right)$, pD9 $\left(P_{\text {reg }} / P_{\text {out }} /\right.$ RBS $_{\text {out }}$ pattern at level $\left.+1 /-1 /+1\right)$, pD10 $\left(P_{\text {reg }} / P_{\text {out }} /\right.$ RBS $_{\text {out }}$ pattern at level $\left.-1 / 0 /+1\right)$, pD11 $\left(P_{\text {reg }} / P_{\text {out }} /\right.$ RBS $_{\text {out }}$ pattern at level $\left.+1 /+1 /-1\right)$, pD12 $\left(P_{\text {reg }} / P_{\text {out }} /\right.$ RBS $_{\text {out }}$ pattern at level $-1 /$ $1 /+1)$, and $\mathrm{pD} 13\left(P_{\text {reg }} / P_{\text {out }} / \mathrm{RBS}_{\text {out }}\right.$ pattern at level $\left.-1 /+1 /-1\right)$. Validation constructs for modelling of the DoE dataset were created with in vivo assembly using selected members of the $P_{\text {reg }}$ 
library linearized by inverse PCR (primers AB10/128) and pcaV amplified by PCR (primers $A B 96 / 127$ ) from p44-pcaV. The pKIKO set of vectors (6) was used to make genomic insertions of different $P A B$ variants. The PAB was amplified from selected DoE plasmids by PCR with primers $A B 101 / 102$ and inserted via in vivo assembly into pKIKOarsBKm that had been linearized by inverse PCR with primers AB29/30.

For the ferulic acid biosensor ( $F A B$ ) designs, the pFABsP vector was constructed by isothermal assembly, using (i) pET28a (Novagen) served as a backbone and linearized by PCR with primers FAB1/2 to remove lacl and the T7 promoter; (ii) the chimeric $P_{\mathrm{LC}}$ promoteroperator (7) and the G10 RBS were incorporated into the forward primer of the FAB3/4 pair and used to amplify sfGFP from a synthetic gene (IDT) and (iii) the FerC transcription factor and FerA enzyme (7) amplified with primers FAB5/6 from p15FABs to yield pFABsP. The new strong promoter-operator $\mathrm{P}_{\mathrm{LC} 2}$ (Supplementary Figure 3 ) was synthesised as a gBlock (IDT) and exchanged with the $P_{\mathrm{LC}}$ promoter by isothermal assembly using $\mathrm{pFABs} P_{\mathrm{LC}}$ linearized by inverse PCR with primers FAB6/7.

The plasmids for DoE pFABs1 $\left(P_{\text {regc }} / P_{\text {enzA }} /\right.$ RBS $_{\text {out }}$ pattern at levels $\left.-1 /-1 /+1\right)$ to pFABs9 $\left(P_{\text {regc }}\right.$ $I P_{\text {enzA }} /$ RBS $_{\text {out }}$ pattern at levels $+1 /+1 /+1$ ) were generated using the $\mathrm{pFABsP} \mathrm{P}_{\mathrm{LC} 2}$ as backbone. The constructs were made by isothermal assembly using four PCR products as parts: (i) The backbone with ferA_p28 (ColE1)_P $\mathrm{LC2}_{2}$ G10_sfGFP was linearized from pFABsP $\mathrm{LC2}_{2}$ with primers FAB9/10, (ii) Promoters corresponding to levels $-1,0$ and +1 from the $P_{\text {reg library (B20, }}$ lo $\mathrm{B} 10$ and B12, respectively) amplified with primers FAB11/12 to be placed upstream to ferC (renamed as $P_{\text {regc }}$ promoters), (iii) ferC amplified with primers FAB13/14 from p15ferCA (7),

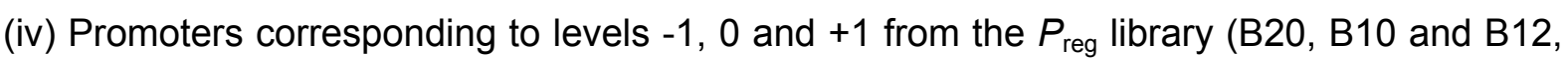
respectively) amplified with primers $\mathrm{FAB} 15 / 16$ to be placed upstream of ferA (renamed as $P_{\text {enzA }}$ promoters). Plasmids lacking ferC (pFABsPLC2 FerC KO) or ferA (pFABsPLC2 FerA

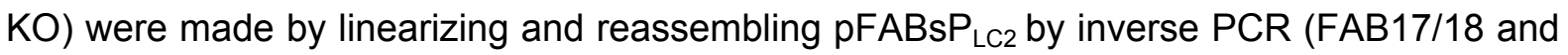
FAB19/20, respectively).

The plasmids for the second iteration pFABsG12 $\left(P_{\text {regc }} / P_{\text {enzA }} /\right.$ RBS $_{\text {out }}$ pattern at levels $+1 /+1 /+0.81)$, pFABsG19 $\left(P_{\text {regc }} / P_{\text {enzA }} /\right.$ RBS $_{\text {out }}$ pattern at levels $\left.+1 /+1 /+0.89\right)$ and pFABsG21 $\left(P_{\text {regc }} / P_{\text {enzA }} /\right.$ RBS $_{\text {out }}$ pattern at levels $\left.+1 /+1 /+0.94\right)$ were generated using $\mathrm{pFABs} 9$ as backbone. Forward primers (FAB21, FAB22, FAB23) were designed with the sequences from the $\mathrm{RBS}_{\text {out }}$ library corresponding to levels $0.81,0.89$ and 0.94 . A reverse primer (FAB24) with overlapping nucleotides to the forward primers was designed. Inverse PCR of pFABs9 with these primers followed by isothermal assembly was carried out to insert the new RBS sequences. 
The pcaK gene from Pseudomonas putida was synthetized (IDT) with codon-optimsation for expression in $E$. coli with a short translational initiation region (AGGAGGAAAAAAA) at the 5' of the start codon. The gene was inserted downstream of pcaV via in vivo assembly into plasmid p131C-B10, linearised by PCR with primers AB10/167, to create p131C-B10-pcaK. The extender plasmid p261-lacl-pcaK, contains the p15A origin and a kanamycin selection marker, and was assembled by isothermal assembly from the following fragments: (i) the pSEVA261 backbone and linearized by inverse PCR with primers AB9/10; (ii) the lacl gene amplified by PCR from pET44 with primers AB197/198; (iii) the $P_{\mathrm{pv}}$ promoter, G10 RBS (set at level 0) and a 150 bp spacer amplified by PCR with primers AB195/196; and (iv) synthetic DNA (IDT) consisting of the $P_{\text {Llac01 }}$ promoter (8) and G10 RBS (level -1). The additional combinatorial RBS constructs were constructed by isothermal assembly using ssDNA oligonucleotides (IDT) (AB 303-309) into the p261-lacl-pcaK backbone, linearized by inverse PCR with primers $A B 301 / 302$.

Benchmarking plasmids were constructed by in vivo assembly. pET44 and pBAD were linearised by inverse $P C R$ with primers $A B 159 / 160$ and $A B 163 / 164$, respectively. sfGFP was amplified from p131B with primers AB161/162 for insertion into pET44 and AB165/166 for insertion into pBAD. pCK302 was a gift from John Heap (Addgene plasmid \#87768). 


\section{SUPPLEMENTARY FIGURES}
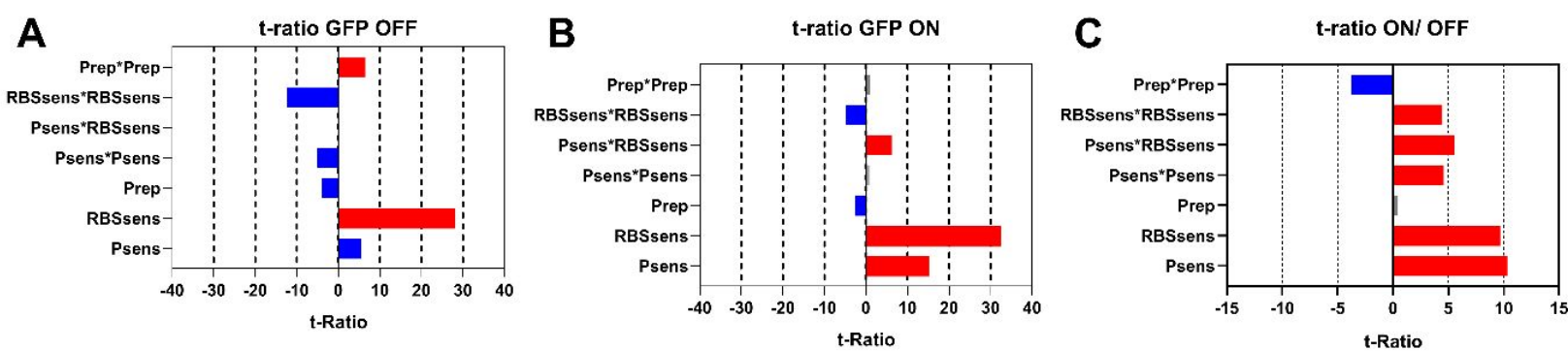

\section{Supplementary Figure 1. Factor screening and selection}

(A-C) Lenth t-ratio of each factor, (A) OFF, (B) ON, and (C) ON/ OFF, showing those factors deemed important by the JMP factor screening platform. The t-ratio is derived from the PSE (OFF PSE $=38.2239$, ON PSE $=206.038$, ON $/$ OFF PSE $=0.71191$ ) and is used to assess factor importance. The colour of the bar indicates the predicted effect of this factor on the indicated response (blue - negative, red - positive). Factors deemed significant at the 0.1 confidence level are deemed significant and were included in the model. 
A

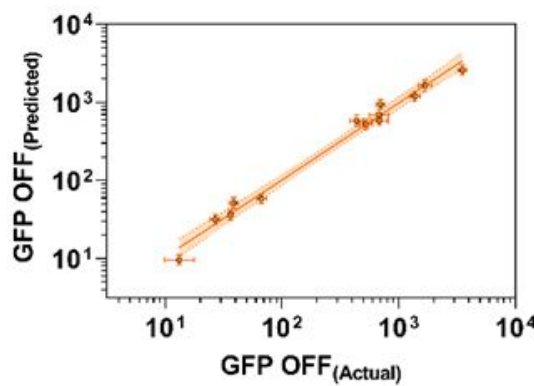

B

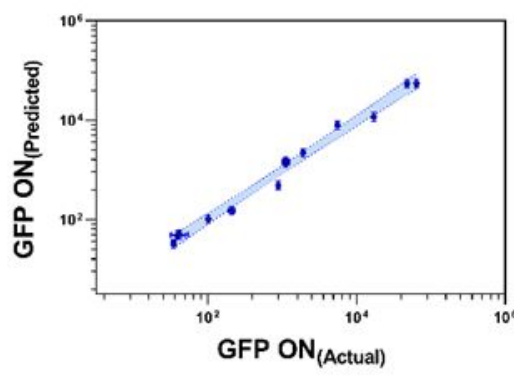

C

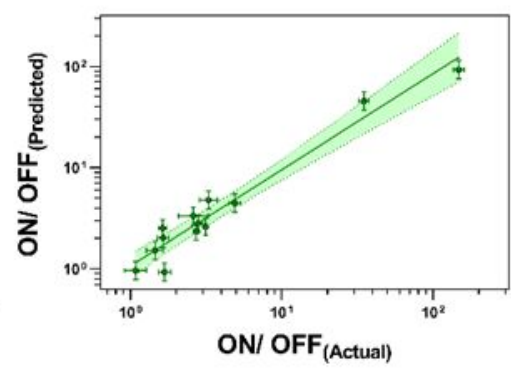

Supplementary Figure 2. Least Squares model performance

Actual versus predicted plots showing the performance of the Least Squares regression model in predicting (A) OFF, (B) ON and (C) ON/OFF. The model shows good prediction of all three responses. OFF $\mathrm{R}^{2}=0.986, P=1.2 \times 10^{-11}$, ON R $\mathrm{R}^{2}=0.988, P=6.8 \times 10^{-12}$, ON/ OFF R $R^{2}=0.95, P=1.6 \times 10^{-08}$. 


\section{GCATGCTATGCTATGGCTTATAGCATTTGACA ATGCTATGGCTTATAGCATGATACTGAGCACATCAGCA \\ GGACGCACTGACCGATTTAACTTTAAGAAGGAGATATACATatg..}

1. $P_{L C}$

$-35$

$-10$

GCATGCAATGCTATGGCTTATAGCATTTGACAGCTAGCTCAGTCCTAGGTATGCTATGGCTTATAGCATG 2. $P_{\mathrm{LC} 2}$

ACGCACTGACCGATTTAACTTTAAGAAGGAGATATACATatg.

*Sphingobium Operator : (19 bp) IR2: $\underline{\text { ATGCTATGGCTTATAGCAT }}$

Supplementary Figure 3. Reengineering of the promoter-operator for the FA Biosensor.

The original promoter-operators $P_{L C}(1)$ and new reengineered $P_{L C 2}$ (2) sequences downstream to a 5' prime region and the Rogers G10 RBS (orange) followed by a sfGFP gene are shown. The IR2 palindromic DNA operator sequence from Sphingobium (light blue) is also shown. The promoter $P_{L C 2}$ was designed replacing the -35 region of the Phage lambda promoter $\left(P_{L}\right)$ for IR2 and fusing it with the spacer sequence of the strong constitutive promoter from the Anderson's library (BBa_J23119). 


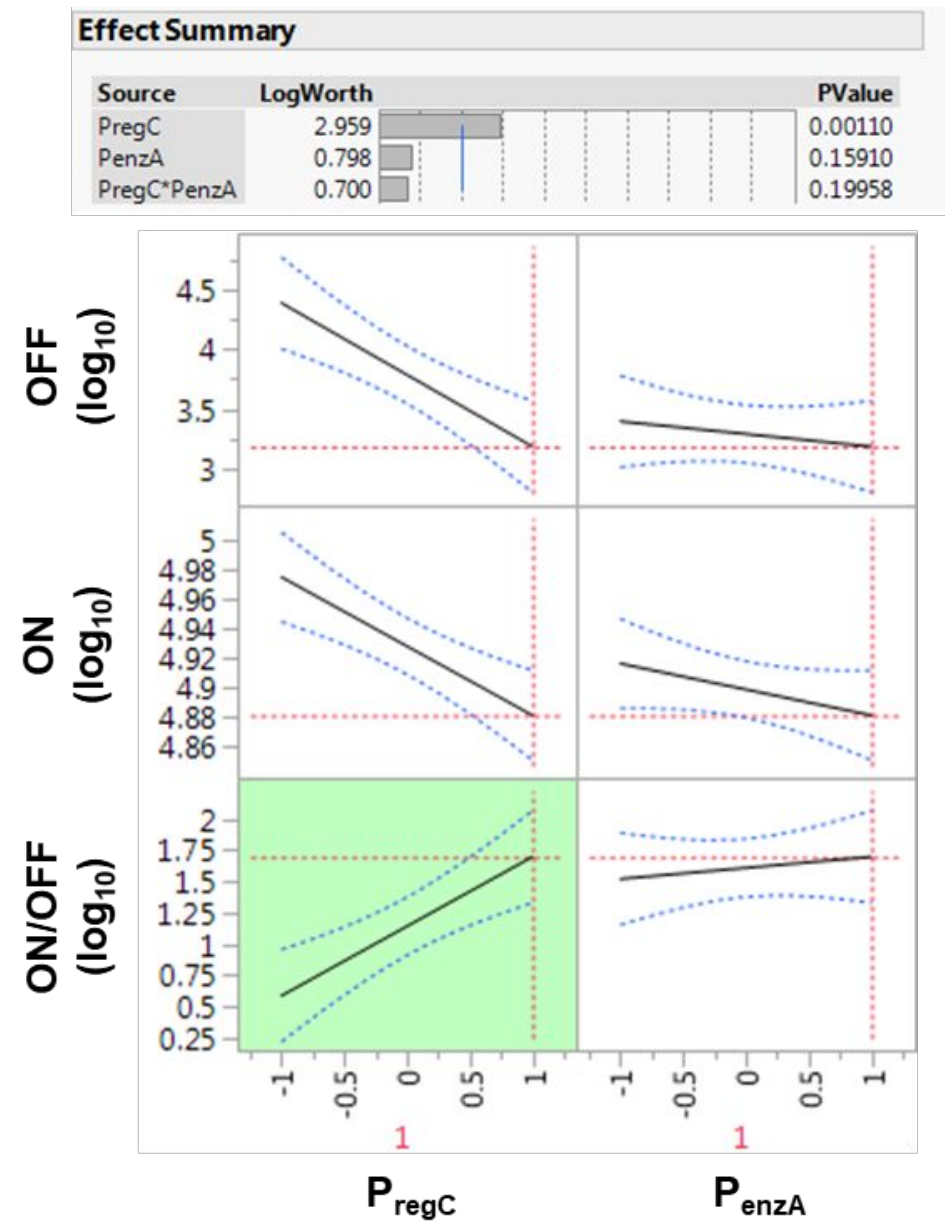

\section{Supplementary Figure 4. Full factorial DoE model for FAB.}

Standard least squares regression (SLSR) model of the DoE dataset. Effect summary of $P_{\text {regc }}, P_{\text {enzA }}$ and $P_{\text {regc }}{ }^{*} P_{\text {enzA }}$ for OFF, ON and ON/OFF showing significative effect of $P_{\text {regc }}$ $(P<0.05)$ for the performance. Model prediction of $P_{\text {regc }}$ and $P_{\text {enzA }}$ for OFF, ON and ON/OFF showing positive linear effect of $P_{\text {regc }}$ levels for ON/OFF (green framed square). 


\section{SUPPLEMENTARY TABLES}

Supplementary Table 1. Raw data for the PAB definitive screening design.

\begin{tabular}{cccccccc|ccc} 
Construct & Trial & $\boldsymbol{P}_{\text {reg }}$ & $\boldsymbol{P}_{\text {out }}$ & RBS $_{\text {out }}$ & \multicolumn{3}{c}{ OFF } & \multicolumn{3}{c}{ ON } \\
pD1 & 1 & 0 & 0 & 0 & 612.7 & 578.4 & 590.6 & 1013.8 & 1046.0 & 1046.6 \\
pD2 & 2 & 0 & 1 & 1 & 398.5 & 394.3 & 400.9 & 61768.3 & 61213.1 & 63230.6 \\
pD3 & 3 & -1 & -1 & -1 & 28.3 & 29.6 & 28.8 & 41.8 & 44.3 & 51.0 \\
pD4 & 4 & 1 & -1 & 0 & 481.0 & 481.0 & 477.5 & 843.2 & 867.3 & 870.9 \\
pD5 & 5 & -1 & 1 & 0 & 1533.6 & 1593.5 & 1502.7 & 5430.4 & 5621.1 & 5587.0 \\
pD6 & 6 & 0 & -1 & -1 & 21.0 & 14.1 & 13.8 & 34.0 & 42.1 & 31.8 \\
pD7 & 7 & 1 & 1 & 1 & 1247.3 & 1322.5 & 1276.7 & 45791.4 & 46571.6 & 49052.4 \\
pD8 & 8 & 1 & 0 & -1 & 36.3 & 40.2 & 46.4 & 46.5 & 51.9 & 50.8 \\
pD9 & 9 & 1 & -1 & 1 & 593.7 & 602.0 & 630.9 & 1035.5 & 1037.7 & 1025.5 \\
pD10 & 10 & -1 & 0 & 1 & 3252.4 & 3255.1 & 3407.2 & 17056.6 & 17312.8 & 17266.8 \\
pD11 & 11 & 1 & 1 & -1 & 32.6 & 37.9 & 42.5 & 96.9 & 101.9 & 101.2 \\
pD12 & 12 & -1 & -1 & 1 & 645.4 & 650.4 & 683.4 & 1753.2 & 1969.2 & 1801.8 \\
pD13 & 13 & -1 & 1 & -1 & 84.2 & 64.5 & 67.1 & 220.5 & 246.6 & 212.7 \\
& & & & & & & & & &
\end{tabular}


Supplementary Table 2. Definitive screening design factor screening.

\begin{tabular}{|c|c|c|c|c|c|c|c|c|c|c|c|c|}
\hline Term & Contrast & $\begin{array}{l}\quad \text { GFF } \\
\text { Lenth } \\
\text { t-Ratio }\end{array}$ & $\begin{array}{l}\text { OFF }(\log 10) \\
\text { Individual } \\
\text { p-value }\end{array}$ & $\begin{array}{l}\text { Simultaneous } \\
\text { p-Value }\end{array}$ & Contrast & $\begin{array}{l}\quad \text { GF } \\
\text { Lenth t- } \\
\text { Ratio }\end{array}$ & $\begin{array}{l}\text { OFF (log10) } \\
\text { Individual } \\
\text { p-value }\end{array}$ & $\begin{array}{l}\text { Simultaneous } \\
\text { p-Value }\end{array}$ & Contrast & $\begin{array}{l}\quad \text { GFF } \\
\text { Lenth t- } \\
\text { Ratio }\end{array}$ & $\begin{array}{l}\text { OFF (log10) } \\
\text { Individual } \\
\text { p-value }\end{array}$ & $\begin{array}{l}\text { Simultaneous } \\
\text { p-Value }\end{array}$ \\
\hline Prep & -293.405 & -11.57 & $1.5 \mathrm{E}-09$ & $1.60341 \mathrm{E}-05$ & 2214.7 & 8.74 & 0.000118247 & 0.000708798 & 2.3319 & 2.33 & 0.033105519 & 0.408426961 \\
\hline Prep*Prep & 223.873 & 8.83 & 3.0372E-06 & 0.000473797 & -2015.4 & -7.95 & 0.000183189 & 0.001025964 & -13.298 & -13.3 & $4 \mathrm{E}-10$ & 2.53E-08 \\
\hline Prep*Psens & -182.802 & -7.21 & 0.000132789 & 0.002035138 & 10014.2 & 39.5 & $<1.0 \mathrm{E}-25$ & $<1.0 \mathrm{E}-25$ & 15.9431 & 15.95 & $<8.5 \mathrm{E}-15$ & $<3.2 \mathrm{E}-11$ \\
\hline Psens & 145.343 & 5.73 & 0.000783357 & 0.007750242 & -996.1 & -3.93 & 0.004264757 & 0.059177616 & -5.1029 & -5.11 & 0.00108595 & 0.014827773 \\
\hline Psens*Psens & -278.853 & -11 & $8.8 \mathrm{E}-09$ & 0.000038746 & 1974.3 & 7.79 & 0.000196565 & 0.001084874 & 7.439 & 7.44 & 0.000206546 & 0.002076476 \\
\hline RBSsens & 575.014 & 22.68 & $<1.0 \mathrm{E}-25$ & $<1.8 \mathrm{E}-16$ & 11588.6 & 45.71 & $<1.0 \mathrm{E}-25$ & $<1.0 \mathrm{E}-25$ & 15.8569 & 15.86 & $<1.1 \mathrm{E}-14$ & $<4.0 \mathrm{E}-11$ \\
\hline RBSsens*Prep & -210.003 & -8.28 & $1.10021 \mathrm{E}-05$ & 0.000640039 & -767.3 & -3.03 & 0.01308755 & 0.183798057 & -7.932 & -7.94 & 0.000151893 & 0.001130195 \\
\hline RBSsens*Prep*Prep & 312.489 & 12.32 & $1 \mathrm{E}-10$ & 4.7395E-06 & 10695.3 & 42.19 & $<1.0 \mathrm{E}-25$ & $<1.0 \mathrm{E}-25$ & 17.3039 & 17.31 & $<6.2 \mathrm{E}-17$ & $<6.6 \mathrm{E}-13$ \\
\hline RBSsens*Prep*Psens & -305.415 & -12.05 & $3 E-10$ & 7.5076E-06 & -1970.9 & -7.77 & 0.000197683 & 0.001089795 & -13.7655 & -13.77 & $1 \mathrm{E}-10$ & 8.5E-09 \\
\hline RBSsens*Psens & 228.542 & 9.01 & $1.9361 \mathrm{E}-06$ & 0.000452371 & 2303.5 & 9.09 & $3.96331 \mathrm{E}-05$ & 0.000462061 & 6.8437 & 6.85 & 0.000295195 & 0.003063257 \\
\hline RBSsens*RBSsens & -116.524 & -4.6 & 0.00141157 & 0.025293161 & 4548.2 & 17.94 & $<3.2 \mathrm{E}-16$ & 0.0000001 & 6.6929 & 6.7 & 0.000413395 & 0.003446297 \\
\hline RBSsens*RBSsens ${ }^{*}$ Prep & 41.775 & 1.65 & 0.103189081 & 0.853679627 & 3330.2 & 13.14 & $5.8 \mathrm{E}-09$ & 0.000132891 & 10.3242 & 10.33 & $9.099 \mathrm{E}-07$ & 1.16639E-05 \\
\hline
\end{tabular}


Supplementary Table 3. Parameter estimates for standard least squares model.

\begin{tabular}{|c|c|c|c|c|c|c|c|c|c|c|c|c|}
\hline \multirow[b]{2}{*}{ Term } & \multicolumn{4}{|c|}{ GFP OFF $(\log 10)$} & \multicolumn{4}{|c|}{ GFP ON $(\log 10)$} & \multicolumn{4}{|c|}{ GFP ON/OFF $(\log 10)$} \\
\hline & Estimate & Std Error & t Ratio & Prob $>|t|$ & Estimate & Std Error & t Ratio & Prob $>|t|$ & Estimate & Std Error & t Ratio & Prob $>\mid t$ \\
\hline Intercept & 2.840341 & 0.071618 & 39.66 & $<.0001$ & 3.144749 & 0.091299 & 34.44 & $<.0001$ & 0.304408 & 0.097342 & 3.13 & $0.005 \varepsilon$ \\
\hline Psens & 0.144009 & 0.026528 & 5.43 & $<.0001$ & 0.516504 & 0.033819 & 15.27 & $<.0001$ & 0.372495 & 0.036057 & 10.33 & $<.0001$ \\
\hline RBSsens & 0.748324 & 0.026528 & 28.21 & $<.0001$ & 1.097718 & 0.033819 & 32.46 & $<.0001$ & 0.349394 & 0.036057 & 9.69 & $<.0001$ \\
\hline Prep & -0.10328 & 0.026528 & -3.89 & 0.0011 & -0.08711 & 0.033819 & -2.58 & 0.019 & 0.01617 & 0.036057 & 0.45 & 0.6592 \\
\hline Psens*Psens & -0.28425 & 0.056222 & -5.06 & $<.0001$ & 0.063164 & 0.071673 & 0.88 & 0.3898 & 0.347418 & 0.076417 & 4.55 & 0.0003 \\
\hline Psens*RBSsens & 0.011279 & 0.033663 & 0.34 & 0.7415 & 0.266777 & 0.042913 & 6.22 & $<.0001$ & 0.255498 & 0.045754 & 5.58 & $<.0001$ \\
\hline RBSsens*RBSsens & -0.69105 & 0.056222 & -12.29 & $<.0001$ & -0.3521 & 0.071673 & -4.91 & 0.0001 & 0.338954 & 0.076417 & 4.44 & 0.0003 \\
\hline Prep*Prep & 0.413538 & 0.063683 & 6.49 & $<.0001$ & 0.086261 & 0.081184 & 1.06 & 0.302 & -0.32728 & 0.086558 & -3.78 & 0.0014 \\
\hline
\end{tabular}


Supplementary Table 4. Tuning the PAB for optimal performance by varying the level of $P_{\text {reg }}$ controlling pcaV.

$\begin{array}{llllllll}\text { Construct } & \text { Set } & \boldsymbol{P}_{\text {reg }} & \boldsymbol{P}_{\text {out }} \text { RBS }_{\text {out }} \text { OFF } & \text { ON } & \text { ON/OFF } \\ \text { p131C-B20 } & \text { validation } & -1.00 & 1 & 1 & 14705.3 \pm 430.2 & 69296.2 \pm 407.9 & 4.7 \pm 0.15 \\ \text { p131C-B9 } & \text { validation } & -0.56 & 1 & 1 & 1418.5 \pm 43.9 & 66255.0 \pm 1099.0 & 46.7 \pm 1.6 \\ \text { p131C-B3 } & \text { validation } & -0.28 & 1 & 1 & 816.9 \pm 14.5 & 62160.1 \pm 984.2 & 76.1 \pm 0.57 \\ \text { pD2 } & \text { training } & 0 & 1 & 1 & 397.9 \pm 3.4 & 62070.6 \pm 1042.1 & 156.0 \pm 1.5 \\ \text { p131C-B10 } & \text { validation } & 0.14 & 1 & 1 & 187.3 \pm 0.7 & 51858.5 \pm 507.6 & 276.8 \pm 3.34 \\ \text { p131C-B6 } & \text { validation } & 0.36 & 1 & 1 & 170.8 \pm 1.6 & 39229.3 \pm 796.1 & 229.7 \pm 6.79 \\ \text { p131C-B18 } & \text { validation } & 0.67 & 1 & 1 & 338.2 \pm 7.2 & 20486.1 \pm 166.1 & 60.6 \pm 1.23 \\ \text { pD7 } & \text { training } & 1 & 1 & 1 & 1282.1 \pm 37.9 & 47138.5 \pm 1702.8 & 36.8 \pm 1.6\end{array}$

OFF and ON measurements were made in the absence or presence of $1 \mathrm{mM}$ PCA, respectively. The values for OFF, ON and OFF/ON indicate the mean of three biological replicates with \pm denoting the standard deviation of those replicates. 
Supplementary Table 5. Comparison of the original and optimised PAB.

$\begin{array}{lcccc}\text { PCA biosensor } & \text { OFF } & \text { ON } & \text { ON/OFF } & \text { EC }_{50}(\mu \mathrm{M}) \\ \text { original } & 7.5 \pm 8.0 & 3121.2 \pm 88.4 & 417.4 \pm 95.9 & 537 \\ \text { p131C-B10 } & 186.5 \pm 5.6 & 97099.3 \pm 612.4 & 521.1 \pm 18.9 & 897\end{array}$

The titration was carried out with a PCA concentration ranging from to 3.9 to $4000 \mu \mathrm{M}$. OFF and ON measurements were made in the absence or presence of $4 \mathrm{mM} P C A$, respectively. The values for OFF, ON and OFF/ON indicate the mean of three biological replicates with \pm denoting the standard deviation of those replicates. 
Supplementary Table 6. Tuning the chromosome-integrated PAB for optimal performance by varying the level of $P_{\text {reg }}$ controlling pcaV.

$\begin{array}{lcccccc}\text { Construct } & \boldsymbol{P}_{\text {reg }} & \boldsymbol{P}_{\text {out }} & \text { RBS }_{\text {out }} & \text { OFF } & \text { ON } & \text { ON/OFF } \\ \text { pDK-B9 } & -0.56 & 1 & 1 & 6562.6 \pm 62.4 & 7163.7 \pm 38.4 & 1.09 \pm 0.02 \\ \text { pDK-B20 } & 0.00 & 1 & 1 & 2826.0 \pm 92.8 & 7066.3 \pm 43.8 & 2.50 \pm 0.09 \\ \text { pDK-B10 } & 0.14 & 1 & 1 & 2543.5 \pm 16.6 & 6841.8 \pm 97.9 & 2.69 \pm 0.05 \\ \text { pDK-B6 } & 0.36 & 1 & 1 & 688.7 \pm 24.3 & 6628.1 \pm 83.8 & 9.63 \pm 0.37 \\ \text { pDK-B17 } & 0.53 & 1 & 1 & 357.5 \pm 2.8 & 7071.9 \pm 87.4 & 19.78 \pm 0.25 \\ \text { pDK-B15 } & 0.61 & 1 & 1 & 155.9 \pm 3.0 & 6677.9 \pm 191.8 & 42.85 \pm 1.53 \\ \text { pDK-B18 } & 0.67 & 1 & 1 & 205.6 \pm 6.9 & 7200.8 \pm 135.6 & 35.05 \pm 1.29 \\ \text { pDK-B23 } & 0.77 & 1 & 1 & 264.1 \pm 2.1 & 6721.3 \pm 140.4 & 25.44 \pm 0.35 \\ \text { pDK-B16 } & 0.94 & 1 & 1 & 284.2 \pm 9.6 & 6910.2 \pm 135.6 & 24.34 \pm 1.23 \\ \text { pDK-B12 } & 1.00 & 1 & 1 & 5759.6 \pm 117.7 & 6956.9 \pm 71.4 & 1.21 \pm 0.03\end{array}$

OFF and ON measurements were made in the absence or presence of $1 \mathrm{mM} P C A$, respectively. The values for OFF, ON and OFF/ON indicate the mean of three biological replicates with \pm denoting the standard deviation of those replicates. 
Supplementary Table 7. Comparison of the sensitivity of the PAB to PCA with and without the PcaK transporter.

$\begin{array}{lcccc}\text { PCA biosensor } & \text { OFF } & \text { ON } & \text { ON/OFF } & \text { EC }_{50}(\mu \mathrm{M}) \\ \text { p131C-B10 } & 164.1 \pm 4.5 & 72521.7 \pm 1656.3 & 442.1 \pm 13.2 & 557 \\ \text { p131C-B10-pcaK } & 359.7 \pm 11.7 & 68864.9 \pm 1133.7 & 191.6 \pm 6.4 & 0.335\end{array}$

The titration was carried out with a PCA concentration ranging from to 0.0038 to $4000 \mu \mathrm{M}$. OFF and ON measurements were made in the absence or presence of $4 \mathrm{mM} \mathrm{PCA}$, respectively. The values for OFF, ON and OFF/ON indicate the mean of three biological replicates with \pm denoting the standard deviation of those replicates. 
Supplementary Table 8. Assessment of the performance of dose-response extender variants.

\begin{tabular}{|c|c|c|c|c|c|c|}
\hline $\begin{array}{l}\text { Dose-response } \\
\text { extender }\end{array}$ & OFF & ON & ON/OFF & $\begin{array}{c}\text { Hill } \\
\text { coefficient }\end{array}$ & $\begin{array}{r}\text { EC50 } \\
(\mu \mathrm{M})\end{array}$ & DRLR \\
\hline reporter only & $\begin{array}{l}181.5 \\
\pm 10.3\end{array}$ & $\begin{array}{l}81004.9 \pm \\
356.6\end{array}$ & $447.2 \pm 24.1$ & $0.980 \pm 0.041$ & $281.8 \pm 24.5$ & 88.7 \\
\hline PcaK_-1_Lacl_-1 & $\begin{array}{l}302.7 \\
\pm 27.3\end{array}$ & $\begin{array}{l}104898.5 \pm \\
1063.7\end{array}$ & $348.3 \pm 29.9$ & $1.65 \pm 0.33$ & $1.73 \pm 0.19$ & 14.4 \\
\hline PcaK_-1_Lacl_0 & $\begin{array}{l}210.2 \\
\pm 42.2\end{array}$ & $\begin{array}{l}79030.1 \pm \\
22808.3\end{array}$ & $374.3 \pm 79$ & $0.926 \pm 0.29$ & $11.5 \pm 4.9$ & 117.8 \\
\hline PcaK_0_Lacl_-1 & $\begin{array}{l}417.0 \\
\pm 51.8\end{array}$ & $\begin{array}{l}105094.9 \pm \\
4218.8\end{array}$ & $254.2 \pm 3$ & $1.58 \pm 0.14$ & $0.948 \pm 0.067$ & 16. 3 \\
\hline PcaK_0_Lacl_0 & $\begin{array}{l}263.1 \\
\pm 12.4\end{array}$ & $\begin{array}{l}99438.9 \pm \\
2299.6\end{array}$ & $378.6 \pm 23.4$ & $1.42 \pm 0.089$ & $1.94 \pm 0.09$ & 22.1 \\
\hline PcaK_1_Lacl_-1 & $\begin{array}{l}382 \pm \\
15.2\end{array}$ & $\begin{array}{l}105063.1 \pm \\
5121.3\end{array}$ & $275.3 \pm 16.0$ & $1.79 \pm 0.4$ & $0.354 \pm 0.02$ & 11.7 \\
\hline PcaK_1_Lacl_0 & $\begin{array}{l}170.6 \\
\pm 6.8\end{array}$ & $\begin{array}{l}102360.6 \pm \\
155.3\end{array}$ & $600.6 \pm 24.8$ & $1.69 \pm 0.12$ & $1.65 \pm 0.059$ & 13.6 \\
\hline
\end{tabular}

The titration was carried out with a PCA concentration ranging from to 0.0128 to $1000 \mu \mathrm{M}$. OFF and ON measurements were made in the absence or presence of $1 \mathrm{mM} P C A$, respectively. The values for OFF, ON and OFF/ON indicate the mean of three biological replicates with \pm denoting the standard deviation of those replicates. 
Supplementary Table 9. Comparison of dynamic range of the PAB against popular expression systems.

$\begin{array}{lcc}\begin{array}{l}\text { Expression } \\ \text { system }\end{array} & \text { ON/OFF } 3 \mathbf{h} & \text { ON/OFF } 24 \mathbf{h} \\ P_{\text {araBAD }} / \text { AraC } & 178.5 \pm 12.2 & 36.3 \pm 1.3 \\ P_{\text {pv }} / \text { PcaV } & 224.5 \pm 2.9 & 219.2 \pm 12.0 \\ P_{\text {lac }} / \text { Lacl/T7RNAP } & 363.2 \pm 25.6 & 84.0 \pm 1.0 \\ P_{\text {rhaBAD }} / \text { RhaS } & 27.4 \pm 1.8 & 2.9 \pm 0.2\end{array}$

The following inducers were used: L-arabinose for $P_{\text {araBAD }} /$ AraC; PCA for $P_{\text {pv }} /$ PcaV; IPTG for $P_{\text {lac }} /$ Lacl/T7RNAP; and L-mannose for $P_{\text {rhaBAD }} /$ RhaS. Titrations were carried out with inducers at concentrations ranging from to 3.9 to $4000 \mu \mathrm{M}$. OFF and ON measurements were made in the absence or presence of $4 \mathrm{mM}$ of inducer, respectively. The values for OFF/ON indicate the mean of three biological replicates with \pm denoting the standard deviation of those replicates. 
Supplementary Table 10. Raw data for the FAB full factorial design.

\begin{tabular}{cccccccc|c} 
Construct & Trial & $\boldsymbol{P}_{\text {regc }}$ & $\boldsymbol{P}_{\text {enzA }}$ & RBS $_{\text {out }}$ & \multicolumn{3}{c}{ OFF } & ON \\
pFABs1 & 1 & -1 & -1 & 1 & 14966 & 15030.4 & 14469.1 & 10255893161.893772 .3 \\
pFABs2 & 2 & -1 & 0 & 1 & 8262.1 & 7285.6 & 7940.3 & 91256.486897 .794597 .5 \\
pFABs3 & 3 & -1 & 1 & 1 & 33529.833274 .8 & 33699.2 & 90905.394533 .295824 .9 \\
pFABs4 & 4 & 0 & -1 & 1 & 6846.6 & 6608.7 & 6492.1 & 89215.487395 .590105 .1 \\
pFABs5 & 5 & 0 & 0 & 1 & 6769.1 & 6876.2 & 6683.6 & 88137.986719 .389006 .7 \\
pFABs6 & 6 & 0 & 1 & 1 & 6552.8 & 6517 & 6039.9 & 88549.289600 .488144 .6 \\
pFABs7 & 7 & 1 & -1 & 1 & 2076.8 & 2164.6 & 2180.1 & 79578.685037 .884313 .2 \\
pFABs8 & 8 & 1 & 0 & 1 & 1971.8 & 2042.2 & 1868.6 & 75845.878895 .376475 .9 \\
pFABs9 & 9 & 1 & 1 & 1 & 1356.1 & 1396.5 & 1545.8 & 71633.677672 .775372 .2
\end{tabular}


Supplementary Table 11. Tuning the FAB for optimal dynamic range by varying the level of RBS $_{\text {out }}$ controlling the sfGFP output.

$\begin{array}{lcccccc}\text { Construct } & \boldsymbol{P}_{\text {regc }} & \boldsymbol{P}_{\text {enzA }} & \text { RBS }_{\text {out }} & \text { OFF } & \text { ON } & \text { ON/OFF } \\ \text { pFABs9 } & 1 & 1 & 1 & 83845.3 \pm 2968.9 & 1378.4 \pm 13.7 & 60.8 \pm 2.0 \\ \text { pFABsG21 } & 1 & 1 & 0.94 & 76569.2 \pm 2157.5 & 1018.2 \pm 33.8 & 75.2 \pm 0.8 \\ \text { pFABsG19 } & 1 & 1 & 0.89 & 62005.7 \pm 2732.2 & 666.2 \pm 20.4 & 93.1 \pm 4.0 \\ \text { pFABsG12 } & 1 & 1 & 0.81 & 30783.2 \pm 1224.7 & 261.5 \pm 11.3 & 117.7 \pm 8.4\end{array}$

OFF and ON measurements were made in the absence or presence of $1 \mathrm{mM} \mathrm{FA}$, respectively. The values for OFF, ON and OFF/ON indicate the mean of three biological replicates with \pm denoting the standard deviation of those replicates. 
Supplementary Table 12. Primer used in this study.

\section{Primer Sequence}

name

AB 09 GCGGCCGCGTCGTGACTGGGAAAA

AB 10 GGCCTAGGCGGCCTCCTGTGTGAAATTG

AB 11 AGCGGATAACAATTTCACACAGGA

AB 12 CGCCAGGGTTTTCCCAGTCA

AB 15 AACAATTTCACACAGGAGGCCGCCTAGGCCTTATTTATACAGTTCGTCCATACCGC

AB 18 CCAGGGTTTTCCCAGTCACGACGCGGCCGCTTATTTATACAGTTCATCCATACCATG GG

AB 27 ATGAGCAAAGGTGAAGAACTGTTTAC

AB 28 ATGGTTTCTAAAGGTGAAGAAGAC

AB 29 GCTCGGATCCACTAGTAGAGG

AB 30 CGGTACCGCATGCAATCAT

AB 34 GAAAGTACGTGCAGCCAGAG

AB 39 CCAAATCGCAGCCAATCACA

AB 40 GGTTATCTGGCAGCCGAAAG

AB 61 GAATCCAGAAAAGCGGCCAT

AB 94 AGTCAACACTCTTTTTGATAAATTTTGCATGC

AB 95 GCATGCAAAATTTATCAAAAAGAGTG

AB 100 GGAATTCCATATGTTTTATCCTCTACTAGTTTATTTTTGACACCAGACCAACTGGTAAT $\mathrm{G}$

AB 101 GgAGgATATTCATATAGACCATGATTGCATAGCGGATAACAATTTCACACAGGA

AB 114 CATGCAAAATTTATCAAAAAGAGTGTTGACTATACTCAGTGCCCTGACTATGATACTTA GATTCATACTCAGTGCCCTGACTATTTTAACTTTAAGANNNNNNTATACATATGAGCA AAGGTGAAGAACTGTTTACCG

AB 115 CATGCAAAATTTATCAAAAAGAGTGTTNANNATACTCAGTGCCCTGACTATNATNNTT AGATTCATACTCAGTGCCCTGACTATTTTAACTTTAAGAAGGAGATATACATATGAGC AAAGGTGAAGAACTGTTTACCG

AB 127 GTTTAACTTTGAAATAAGGAGGTAATACAAATGGCAGCAGTTGATCTGGCAAC

AB 128 TTGTATTACCTCCTTATTTCAAAGTTAAAC

AB 129 CCATCGGAAGCTGTGGTATG

AB 130 GATTTACGACCTGCACAGCC

AB 142 CATGCAAAATTTATCAAAAAGAGTGTTCATGATACTCAGTGCCCTGACTATAATGATTA GATTCATACTCAGTGCCCTGACTATTTTAACTTTAAGATAGTCATATACATATGAGCAA AGGTGAAGAACTGTTTACCG

AB 143 CATGCAAAATTTATCAAAAAGAGTGTTCATGATACTCAGTGCCCTGACTATAATGATTA GATTCATACTCAGTGCCCTGACTATTTTAACTTTAAGAAGGGTGTATACATATGAGCA 
AAGGTGAAGAACTGTTTACCG

AB 144

CATGCAAAATTTATCAAAAAGAGTGTTAAAGATACTCAGTGCCCTGACTATTATGTTTA GATTCATACTCAGTGCCCTGACTATTTTAACTTTAAGATAGTCATATACATATGAGCAA AGGTGAAGAACTGTTTACCG

AB 145 CATGCAAAATTTATCAAAAAGAGTGTTAAAGATACTCAGTGCCCTGACTATTATGTTTA GATTCATACTCAGTGCCCTGACTATTTTAACTTTAAGAAGGGTGTATACATATGAGCA AAGGTGAAGAACTGTTTACCG

AB 146 CCACAACGGTTTCCCTCTAC

AB 147 CATAGACCTAGGGCAGCAGA

AB 148 AAAATTATTTGTAGAGGGAAACCGTTGTGGTCTCCCTGAATATANNNTACGAGCCTTA TGCATGCCCGTAAAGTTATCCAGCAACCACTCATAGACCTAGGGCAGCAGATAGGGA CGAC

AB 159 GCCCATATGTATATCTCCTTCTTAAAG

AB 160 TGTTAATTAAGTTGGGCGTTCC

AB 161 GTTTAACTTTAAGAAGgAGATATACATATGAGCAAAGGTGAAGAACTGTTTAC

AB 162 GCCTAGGAACGCCCAACTTAATTAACATTATTTATACAGTTCATCCATACCATGGG

AB 163 CATGGTATATCTCCTTCTTAAAGTTAAAC

AB 164 CTGTTTTGGCGGATGAGAGA

AB 165 TTTTGTTTAACTTTAAGAAGgAGATATACCATGAGCAAAGGTGAAGAACTGTTTAC

AB 166 CTGAAAATCTTCTCTCATCCGCCAAAACAGTTATTTATACAGTTCATCCATACCATGG $\mathrm{G}$

AB 167 TCAACCCGGTGCAACTGC

AB 195 CATATGTATACACCCTTCTTAAAGTTAAA

AB 196 GGCAAAAAACATTATCCAGAACG

AB 197 TTTAACTTTAAGAAGgGTGTATACATATGGTGAAACCAGTAACGTTATACGATG

AB 198 CCAGGGTTTTCCCAGTCACGACGCGGCCGCTCACTGCCCGCTTTCCAG

AB 199 ACGTCTAAGCTTTTACCTACAGAATTTTGCGCCTGATTCATATGTATATGACTATCTTA AAGTTAAAGGTCAGTGCGTCCTGCTGATGTGCTCAGTATCTTGTTATCCGCTCACAAT GTCAATTGTTATCCGCTCACAATTCTCGAGGGCAAAAAACATTATCCAGAACGGGAGT GCGCC

FAB 1 GGCCGATTCATTAATGCAGCTGACGCAATTAATGTAAGTTAGCT

FAB 2 GATGATTTCTCGGTACCGCATGTAACAAAGCCCGAAAGGAAG

FAB 3 AGCTTCCTTTCGGGCTTTGTTACATGCGGTACCGAGAAATCATC

FAB 4 CTTCCGATGGCTGCCTGACGCCAGTAGTAGGTTGAGGCCGTT

FAB 5 TCAACGGCCTCAACCTACTACTGGCGTCAGGCAGCCATCGGA

FAB 6 AGCTAACTTACATTAATTGCGTCAGCTGCATTAATGAATCGGCCAAC

FAB 7 ATGAGCAAAGGTGAAGAACTGTTTACCG

FAB 8 CTCCCGTTCTGGATAATGTTTTTTGCC

FAB 9 CTTTGAAATAAGGAGGTAATACAAATGGCCGTTGAAGCCGGTGTTCGTC 
FAB 10 GGCAAAAAACATTATCCAGAACGGGAGTGCGCC

FAB 11 GCACTCCCGTTCTGGATAATGTTTTTTGCCCACAGCTAACACCACGTC

FAB 12 GATCATCCTGACGCATACGTTCACCCATTTGTATTACCTCCTTATTTCAAAGTTA

FAB 13 TAACTTTGAAATAAGGAGGTAATACAAATGGGTGAACGTATGCGTCAGGATGATC

FAB 14 GATAGGGACGACGTGGTGTTAGCTGTGTCTAGAATAAAACGAAAGGCCCAGTCTTC

FAB 15 GAAGACTGGGCCTTTCGTTTTATTCTAGACACAGCTAACACCACGTCGTCCCTATC

FAB 16 CTGAGGACGAACACCGGCTTCAACGGCCATTTGTATTACCTCCTTATTTCAAAGTTAA $A C$

FAB 17 TCTAGACCATCGAATGGTGCAAAACCTTTCGCG

FAB 18 GGCAAAAAACATTATCCAGAACGGGAGTGCGCC

FAB 19 GGCCGATTCATTAATGCAGCTGACGCAATTAATGTAAGTTAGCT

FAB 20 GTTACTGGTTTCACATTCACCACCC

FAB 21 GATTTAACTTTAAGACTTTGGTATACATATGAGCAAAGGTGAAGAACT

FAB 22 GATTTAACTTTAAGAGGCTTATATACATATGAGCAAAGGTGAAGAACT

FAB 23 GATTTAACTTTAAGAGGGAGGTATACATATGAGCAAAGGTGAAGAACT

FAB 24 CTTAAAGTTAAATCGGTCAGTGCGTCATGC

AB 301 ATGGTGAAACCAGTAACGTTATACGATGTCG

AB 302 ATGAATCAGgCGCAAAATTCTGTAGgTAAAAGC

AB 303 GCTTTTACCTACAGAATTTTGCGCCTGATTCATATGTATACACCCTTCTTAAAGTTAAA GGTCAGTGCGTCCTGCTGATGTGCTCAGTATCTTGTTATCCGCTCACAATGTCAATTG TTATCCGCTCACAATTCTCGAGGGCAAAAAACATTATCCAGAACGGGAGTGCGCCTT GAGCGACACGAATTATGCAGTGATTTACGACCTGCACAGCCATACCACAGCTTCCGA TGGCTGCCTGACGCCAGAAGCATTGGTGCACCGTGCAGTCGATGATAAGCTGTCAA ACGCATGCAAAATTTATCAAAAAGAGTGTTGACTATACTCAGTGCCCTGACTATGATA CTTAGATTCATACTCAGTGCCCTGACTATTTTAACTTTAAGAAGGGTGTATACATATGG TGAAACCAGTAACGTTATACGATGTCG

AB 304 GCTTTTACCTACAGAATTTTGCGCCTGATTCATATGTATATGACTATCTTAAAGTTAAA GGTCAGTGCGTCCTGCTGATGTGCTCAGTATCTTGTTATCCGCTCACAATGTCAATTG TTATCCGCTCACAATTCTCGAGGGCAAAAAACATTATCCAGAACGGGAGTGCGCCTT GAGCGACACGAATTATGCAGTGATTTACGACCTGCACAGCCATACCACAGCTTCCGA TGGCTGCCTGACGCCAGAAGCATTGGTGCACCGTGCAGTCGATGATAAGCTGTCAA ACGCATGCAAAATTTATCAAAAAGAGTGTTGACTATACTCAGTGCCCTGACTATGATA CTTAGATTCATACTCAGTGCCCTGACTATTTTAACTTTAAGAAGGGTGTATACATATGG TGAAACCAGTAACGTTATACGATGTCG

AB 305 GCTTTTACCTACAGAATTTTGCGCCTGATTCATATGTATATCTCCTTCTTAAAGTTAAA GGTCAGTGCGTCCTGCTGATGTGCTCAGTATCTTGTTATCCGCTCACAATGTCAATTG TTATCCGCTCACAATTCTCGAGGGCAAAAAACATTATCCAGAACGGGAGTGCGCCTT GAGCGACACGAATTATGCAGTGATTTACGACCTGCACAGCCATACCACAGCTTCCGA TGGCTGCCTGACGCCAGAAGCATTGGTGCACCGTGCAGTCGATGATAAGCTGTCAA 
ACGCATGCAAAATTTATCAAAAAGAGTGTTGACTATACTCAGTGCCCTGACTATGATA CTTAGATTCATACTCAGTGCCCTGACTATTTTAACTTTAAGAAGGGTGTATACATATGG TGAAACCAGTAACGTTATACGATGTCG

AB 306 GCTTTTACCTACAGAATTTTGCGCCTGATTCATATGTATACACCCTTCTTAAAGTTAAA GGTCAGTGCGTCCTGCTGATGTGCTCAGTATCTTGTTATCCGCTCACAATGTCAATTG TTATCCGCTCACAATTCTCGAGGGCAAAAAACATTATCCAGAACGGGAGTGCGCCTT GAGCGACACGAATTATGCAGTGATTTACGACCTGCACAGCCATACCACAGCTTCCGA TGGCTGCCTGACGCCAGAAGCATTGGTGCACCGTGCAGTCGATGATAAGCTGTCAA ACGCATGCAAAATTTATCAAAAAGAGTGTTGACTATACTCAGTGCCCTGACTATGATA CTTAGATTCATACTCAGTGCCCTGACTATTTTAACTTTAAGATAGTCATATACATATGG TGAAACCAGTAACGTTATACGATGTCG

AB 307 GCTTTTACCTACAGAATTTTGCGCCTGATTCATATGTATACACCCTTCTTAAAGTTAAA GGTCAGTGCGTCCTGCTGATGTGCTCAGTATCTTGTTATCCGCTCACAATGTCAATTG TTATCCGCTCACAATTCTCGAGGGCAAAAAACATTATCCAGAACGGGAGTGCGCCTT GAGCGACACGAATTATGCAGTGATTTACGACCTGCACAGCCATACCACAGCTTCCGA TGGCTGCCTGACGCCAGAAGCATTGGTGCACCGTGCAGTCGATGATAAGCTGTCAA ACGCATGCAAAATTTATCAAAAAGAGTGTTGACTATACTCAGTGCCCTGACTATGATA CTTAGATTCATACTCAGTGCCCTGACTATTTTAACTTTAAGAAGGAGATATACATATGG TGAAACCAGTAACGTTATACGATGTCG

AB 308 GCTTTTACCTACAGAATTTTGCGCCTGATTCATATGTATATGACTATCTTAAAGTTAAA GGTCAGTGCGTCCTGCTGATGTGCTCAGTATCTTGTTATCCGCTCACAATGTCAATTG TTATCCGCTCACAATTCTCGAGGGCAAAAAACATTATCCAGAACGGGAGTGCGCCTT GAGCGACACGAATTATGCAGTGATTTACGACCTGCACAGCCATACCACAGCTTCCGA TGGCTGCCTGACGCCAGAAGCATTGGTGCACCGTGCAGTCGATGATAAGCTGTCAA ACGCATGCAAAATTTATCAAAAAGAGTGTTGACTATACTCAGTGCCCTGACTATGATA CTTAGATTCATACTCAGTGCCCTGACTATTTTAACTTTAAGATAGTCATATACATATGG TGAAACCAGTAACGTTATACGATGTCG

AB 309 GCTTTTACCTACAGAATTTTGCGCCTGATTCATATGTATATCTCCTTCTTAAAGTTAAA GGTCAGTGCGTCCTGCTGATGTGCTCAGTATCTTGTTATCCGCTCACAATGTCAATTG TTATCCGCTCACAATTCTCGAGGGCAAAAAACATTATCCAGAACGGGAGTGCGCCTT GAGCGACACGAATTATGCAGTGATTTACGACCTGCACAGCCATACCACAGCTTCCGA TGGCTGCCTGACGCCAGAAGCATTGGTGCACCGTGCAGTCGATGATAAGCTGTCAA ACGCATGCAAAATTTATCAAAAAGAGTGTTGACTATACTCAGTGCCCTGACTATGATA CTTAGATTCATACTCAGTGCCCTGACTATTTTAACTTTAAGATAGTCATATACATATGG TGAAACCAGTAACGTTATACGATGTCG 
Supplementary Table 13. Plasmid used or constructed in this study

\begin{tabular}{|c|c|c|}
\hline Plasmid name & Relevant characteristics ${ }^{a}$ & $\begin{array}{l}\text { Source or } \\
\text { reference }\end{array}$ \\
\hline pSEVA131 & Cloning vector; oriV (pBBR1), $\mathrm{Am}^{\mathrm{r}}$ & (9) \\
\hline pSEVA 261 & Cloning vector; oriV (p15A), $\mathrm{Km}^{\mathrm{r}}$ & (9) \\
\hline pET28a & Expression vector; $P_{\text {lad }} /$ Lacl/T7RNAP; oriV (pBR322), $\mathrm{Km}^{\mathrm{r}}$ & Merck \\
\hline pET44a & Expression vector; $P_{\text {lad }} /$ Lacl/T7RNAP; oriV (pBR322), Am ${ }^{r}$ & Merck \\
\hline pBAD & Expression vector; $P_{\text {araBAD }} / \mathrm{AraC}$; oriV (pBR322), $\mathrm{Am}^{r}$ & ThermoFisher \\
\hline pCK302 & sfGFP expression vector; $P_{\text {rhaBAD }} /$ RhaS; oriV (pBR322), Am ${ }^{r}$ & $(10)$ \\
\hline pKIKOarsBKm & Integration vector; oriV (RK6), $\mathrm{Am}^{r}, \mathrm{Km}^{r}$ & $(6)$ \\
\hline p131B & Template vector with $m$ Cherry and sfGFP; oriV (pBBR1), Am ${ }^{r}$ & This study \\
\hline $\mathrm{p} 131 \mathrm{~B}-\mathrm{B} X^{\mathrm{b}}$ & 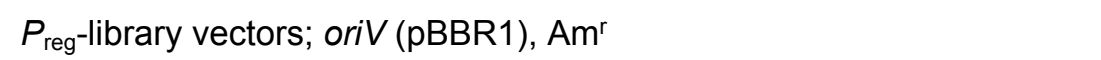 & This study \\
\hline p131B-GXb & RBS $_{\text {out-library vectors; oriV (pBBR1), Am }}{ }^{r}$ & This study \\
\hline $\mathrm{p} 131-\mathrm{V} X^{\mathrm{b}}$ & $P_{\text {out }}$ library vectors; oriV (pBBR1), $\mathrm{Am}^{\mathrm{r}}$ & This study \\
\hline $\mathrm{pDX}$ & DoE PCA biosensor vectors; oriV (pBBR1), Am ${ }^{r}$ & This study \\
\hline p131CB- $X^{b}$ & DoE PCA biosensor validation vectors; oriV (pBBR1), $\mathrm{Am}^{r}$ & This study \\
\hline pDK-BX & DoE PCA biosensor integration vectors; oriV (pBBR1), $\mathrm{Am}^{r}$ & This study \\
\hline pET44-sfGFP & sfGFP expression vector; $P_{\text {lad }} /$ Lacl/T7RNAP; oriV (pBR322), Am & This study \\
\hline pBAD-sfGFP & sfGFP expression vector; $P_{\text {araBAD }} / \mathrm{AraC}$; oriV (pBR322), Amr & This study \\
\hline pFABsP $P_{L C}$ & FA biosensor vector promoter variant $P_{\mathrm{LC}} ;$ oriV (pBR322), $\mathrm{Km}^{r}$ & This study \\
\hline pFABsP $P_{L C 2}$ & FA biosensor vector promoter variant $P_{\mathrm{LC} 2} ;$ oriV (pBR322), $\mathrm{Km}^{\mathrm{r}}$ & This study \\
\hline pFABsP $\mathrm{LC2}_{2}$ FerC KO & FA biosensor vector promoter variant $P_{\mathrm{LC} 2} \Delta f e r C$; oriV (pBR322), $\mathrm{Km}^{\mathrm{r}}$ & This study \\
\hline pFABsP $\mathrm{LC2}_{2}$ FerA KO & FA biosensor vector promoter variant $P_{\mathrm{LC} 2} \Delta$ ferA; oriV (pBR322), $\mathrm{Km}^{\mathrm{r}}$ & This study \\
\hline pFABsX (DOE) & DoE FA biosensor vectors; oriV (pBR322), $\mathrm{Km}^{\mathrm{r}}$ & This study \\
\hline pFABsG12 & DoE FA biosensor variant $\mathrm{RBS}_{\text {out }}$ at 0.81 ; oriV (pBR322), $\mathrm{Km}^{\mathrm{r}}$ & This study \\
\hline pFABsG19 & DoE FA biosensor variant $\mathrm{RBS}_{\text {out }}$ at 0.89 ; oriV (pBR322), $\mathrm{Km}^{\mathrm{r}}$ & This study \\
\hline pFABsG21 & DoE FA biosensor variant $\mathrm{RBS}_{\text {out }}$ at 0.94 ; oriV (pBR322), $\mathrm{Km}^{\mathrm{r}}$ & This study \\
\hline p261_Lacl[X]_PcaK[X] $]^{\natural}$ & ${ }^{b}$ DoE PCA biosensor extender vectors; oriV ( $\left.\mathrm{p} 15 \mathrm{~A}\right), \mathrm{Km}^{\mathrm{r}}$ & This study \\
\hline
\end{tabular}




\section{References}

1. Davis, J.H., Rubin, A.J. and Sauer, R.T. (2011) Design, construction and characterization of a set of insulated bacterial promoters. Nucleic Acids Res., 39, 1131-1141.

2. Rogers, J.K., Guzman, C.D., Taylor, N.D., Raman, S., Anderson, K. and Church, G.M. (2015) Synthetic biosensors for precise gene control and real-time monitoring of metabolites. Nucleic Acids Res., 43, 7648-7660.

3. Machado, L.F.M., Currin, A. and Dixon, N. (2019) Directed evolution of the PcaV allosteric transcription factor to generate a biosensor for aromatic aldehydes. J. Biol. Eng. 13, 91

4. Karig, D.K., Iyer, S., Simpson, M.L. and Doktycz, M.J. (2012) Expression optimization and synthetic gene networks in cell-free systems. Nucleic Acids Res., 40, 3763-3774.

5. Benedetti, I., Nikel, P.I. and de Lorenzo, V. (2016) Data on the standardization of a cyclohexanone-responsive expression system for Gram-negative bacteria. Data Brief, 6, 738744.

6. Sabri, S., Steen, J.A., Bongers, M., Nielsen, L.K. and Vickers, C.E. (2013) Knock-in/Knock-out (KIKO) vectors for rapid integration of large DNA sequences, including whole metabolic pathways, onto the Escherichia coli chromosome at well-characterised loci. Microb. Cell Fact., 12,60 .

7. Machado, L.F. and Dixon, N. (2016) Development and substrate specificity screening of an in vivo biosensor for the detection of biomass derived aromatic chemical building blocks. Chem. Commun. (Camb), 52, 11402-11405.

8. Lutz, R. and Bujard, H. (1997) Independent and tight regulation of transcriptional units in Escherichia coli via the LacR/O, the TetR/O and AraC/I1-12 regulatory elements. Nucleic Acids Res., 25, 1203-1210.

9. Silva-Rocha, R., Martinez-Garcia, E., Calles, B., Chavarria, M., Arce-Rodriguez, A., de Las Heras, A., Paez-Espino, A.D., Durante-Rodriguez, G., Kim, J., Nikel, P.I. et al. (2013) The Standard European Vector Architecture (SEVA): a coherent platform for the analysis and deployment of complex prokaryotic phenotypes. Nucleic Acids Res., 41, D666-675.

10. Kelly, C.L., Liu, Z., Yoshihara, A., Jenkinson, S.F., Wormald, M.R., Otero, J., Estevez, A., Kato, A., Marqvorsen, M.H., Fleet, G.W. et al. (2016) Synthetic chemical inducers and genetic decoupling enable orthogonal control of the rhaBAD promoter. ACS Synth. Biol., 5, 1136-1145. 